\title{
Questes
}

\section{Respect et transgression des règles du jeu dans Le}

Lai d'Ignaure

Mathilde Grodet

\section{(2) OpenEdition}

Journals

\section{Édition électronique}

URL : http://journals.openedition.org/questes/670

DOI : 10.4000/questes. 670

ISSN : 2109-9472

\section{Éditeur}

Les Amis de Questes

\section{Édition imprimée}

Date de publication : 15 février 2010

Pagination : 78-86

ISSN : 2102-7188

\section{Référence électronique}

Mathilde Grodet, «Respect et transgression des règles du jeu dans Le Lai d'Ignaure », Questes [En ligne], 18 | 2010, mis en ligne le 01 janvier 2014, consulté le 19 avril 2019. URL : http:// journals.openedition.org/questes/670 ; DOI : 10.4000/questes.670 


\title{
Respect et transgression des règles du jeu dans Le Lai d'Ignaure
}

\author{
Mathilde GRODET
}

Le Lai d'Ignaure, ou Lai du Prisonnier, écrit au début du XIII ${ }^{\mathrm{e}}$ siècle par un certain Renaus, parfois identifié à Renaut de Beaujeu, est le plus ancien texte français conservé exploitant le motif du cœur mangé ${ }^{1}$. Les récits de cœur mangé racontent la vengeance d'un mari trompé qui, pour punir l'infidélité de son épouse, lui sert à table le cœur de son amant ${ }^{2}$. Par rapport aux autres œuvres reprenant ce motif, le Lai d'Ignaure présente deux particularités : la présence non pas d'une, mais de douze dames, et la nature exacte du met consommé, puisqu'au cœur s'ajoute le sexe du chevalier.

Ignaure est un chevalier vaillant et courtois, mais de petite noblesse. Par amour ou par intérêt, il devient l'amant de douze dames de son

\footnotetext{
${ }^{1}$ Contrairement aux autres lais de la même époque, il n'existe pas d'édition récente de ce texte. L'édition de référence est celle de Rita Lejeune : RENAUT DE BEAUJEU, Le lai d'Ignaure ou du Prisonnier, éd. Rita LeJEune, Bruxelles, Académie Royale de Langue et de Littérature Françaises de Belgique, 1938.

2 Plusieurs études consacrées au motif du cœur mangé sont disponibles. Voir en particulier pour la période médiévale : Denis BERTRAND et Jean-Jacques VINCENSINI, "La vengeance est un plat qui se mange cuit», Bulletin du Groupe de Recherches Sémio-Linguistiques, XVI (1980), p. 30-40 ; Mariella Di MAIO, Le Cour mangé. Histoire d'un thème littéraire du Moyen Âge au XIX siècle, Paris, Presses de l'Université Paris Sorbonne, 2005 ; John E. MATZKE, « The legend of the eaten heart », Modern Language Notes, XXVI (1911), p. 1-8; Henri HaUVETTE, « La $39^{\mathrm{e}}$ nouvelle du Décaméron et la légende du cœur mangé », Romania, XLI (1912), p. 184-205 ; Bernard RIBEMONT, "Le cuer del ventre li as trais : cœur arraché, cœur mangé, cœur envolé : un regard médico-théologique sur quelques thèmes littéraires », Le Cuer au Moyen Âge. Réalité et Senefiance, Senefiance n`30, Aix, CUERMA, 1991, p. 345-362; Luciano Rossi, "Il cuore mistico pasto d'amore: dal Lai Guirun al Decameron », Studi provenzali e francesi 82, Romanica Vulgaria, Quaderni 6, L’Aquila, Japadre, 1983, p. 28-128 ; Jean-Jacques VINCENSINI, «Figure de l'imaginaire et figure du discours. Le motif du cœur mangé dans la narration médiévale ", Le Cuer au Moyen Âge. Réalité et Senefiance, op. cit., p. 441-459. On pourra également lire avec intérêt l'ouvrage de Milad DoueIHI, Histoire perverse du cœur humain, Paris, Le Seuil, 1996.
} 
voisinage. Au cours d'un jeu, celles-ci découvrent la situation et conçoivent un plan pour le punir. Mais Ignaure parvient à les amadouer. Elles le somment toutefois de choisir l'une d'elles comme unique amie. Dès lors, Ignaure prend moins de précautions et cette relation est bientôt découverte. Apprenant leur infortune, les douze maris décident de se venger : le dernier époux trompé enlève Ignaure. Les dames entament alors un jeûne afin qu'on leur dise ce qui est arrivé à leur ancien amant. Les maris agacés choisissent de le mettre à mort et de servir à leurs femmes le cœur et le membre viril du chevalier. Cédant aux pressions de leurs époux, les dames acceptent de s'alimenter. Lorsqu'ils leur révèlent le contenu du plat qu'elles ont mangé, elles se laissent mourir de faim.

Dans l'optique de notre séminaire, nous avons porté notre attention sur la scène de jeu qui constitue l'élément perturbateur du récit ${ }^{3}$. Alors que les douze dames sont réunies lors d'une fête, l'une d'elle propose aux autres de simuler une confession afin que chacune avoue le nom de son amant et qu'ainsi elles puissent savoir laquelle a l'ami le plus prestigieux. Nous pouvons rapprocher cette fausse confession d'autres jeux collectifs d'apprentissage amoureux dans lesquels un cadre contraignant oblige à des révélations d'ordre sentimental. Le jeu du roi qui ne ment est probablement le plus célèbre d'entre eux ${ }^{4}$. Mais ici le respect scrupuleux des règles du jeu révèle la transgression des règles du jeu de l'amour courtois.

\section{Les modalités du jeu}

L'auteur inscrit la scène de la fausse confession dans un contexte festif et ludique. Dès les premiers vers, le cadre spatio-temporel de la scène

\footnotetext{
${ }^{3}$ La scène, relativement longue, correspond aux vers 65 à 225 de l'édition mentionnée.

${ }^{4} \mathrm{Ce}$ jeu, très en vogue au XIII ${ }^{\mathrm{e}}$ siècle, est fréquemment cité dans la littérature. Nous le rencontrons dans des œuvres aussi diverses que Le sentier battu de Jean de Condé ou L'Espinette amoureuse de Jean Froisssart. Voir Ernest LANGLOIS, « Le jeu du roi qui ne ment », Mélanges Chabaneau, Romanische Forschungen, t. 23 (1906), p. 163-173.
} 
est planté. Nous apprenons que le jeu a lieu lors de la fête de la saint Jean (v. 66). Il s'agit d'une date repère fréquente dans les œuvres médiévales : elle marque une rupture fatidique dans le récit, les bouleversements de l'intrigue se superposant au renversement astronomique ${ }^{5}$. La nuit de la saint Jean est en effet la nuit la plus courte de l'année, elle coïncide avec le solstice d'été. Il semble qu'elle ait été, déjà à des dates très anciennes, l'occasion de rituels festifs et de réjouissances ${ }^{6}$. C'est encore aujourd'hui une fête folklorique vivace, sur laquelle est venue se greffer par exemple la fête de la musique. Par ailleurs la fausse confession prend place dans un jardin. Or ce vregier, évoqué au vers 70 , est traditionnellement dans la littérature de cette époque un lieu de détente, souvent même de rencontre amoureuse.

Nous notons une certaine harmonie entre ce cadre et l'état d'esprit des personnages. À la gaîté du lieu et du moment fait écho l'allégresse des femmes. De nombreux termes appartenant au champ lexical du plaisir viennent le souligner: esjoïst v. 67, esbanoier v. 69, reviel, v. 84, lie v. $143 \ldots$ Les esprits sont donc à la fête, ce que confirment, à plusieurs reprises, les mentions des sourires et des rires des dames (v. 104, 196). Ce lien entre l'état intérieur et l'ambiance extérieure apparaît dans les paroles mêmes d'une des dames :

« Plainnes sommes de grant reviel.

N'i a cheli n'aint par amours,

Et molt est envoisiés cis jours. » (v. 84-86)

Cette allégresse générale justifie la mise en place du jeu dont les règles suivent immédiatement ces vers.

Sous-entendant un lien de cause/conséquence, les règles contraignantes sont en effet énoncées dans les vers suivants :

\footnotetext{
${ }^{5}$ Nous la retrouvons par exemple dans Yvain de Chrétien de Troyes, dans Le roman de Tristan de Béroul, dans Le Lai de Lanval de Marie de France...

${ }^{6}$ Cf Philippe Walter, La Mémoire du temps. Fêtes et calendriers de Chrétien de Troyes à La Mort Artu, Paris, Champion, 1989.
} 
«"D'une de nous fasons no prestre ;

Seoir en voist en mi cel estre,

Lés cele ente ki est flourie.

Chascune i voist, et se li dist

Cui ele aimme, en confiession,

$\mathrm{Ne}$ a cui elle a fait le don :

Ensi sarons certainnement

Li quele aimme plus hautement."

Toutes respondent : "Bien a dit.

Nous l'otrïons sans contredit.

Vous meïsmes prestres serés,

Les confiesses escouterés.

Alés seoir dalés cel'ente.” » (v. 87-99)

Il s'agit d'une simple proposition de jeu. Pourtant le choix des temps verbaux (impératif et subjonctif présent dans la proposition de la première dame, futur simple de l'indicatif et impératif présent dans la réponse) souligne la force de la règle qui s'impose aux femmes.

Alors même que le jeu va prendre une tournure désagréable à laquelle elles n'avaient pas songé, les dames vont continuer à respecter les règles qu'elles ont établies. Elles vont s'efforcer de faire une imitation parfaite du rite de la confession. Leurs paroles et leurs gestes restituent ainsi ceux des fidèles. Les expressions empruntées au sacrement de la pénitence sont nombreuses : «A confesse vienc, sire prestres. » (v. 106); «les pechiés / Dont vostre cors est entechiés!»(v. 125-126); «Sire je viench a ademanche » (v. 127) ; «Je vous commanch, en penitanche » (v. 128). Quant aux gestes, deux attitudes sont en particulier remarquables, celle d'une des dames qui «a la destre main [bat] sa coupe» (v. 123) et celle du prêtre qui « se saigne » (v. 156). À ces formules et à cette gestuelle s'ajoute encore l'exigence de vérité rappelée à plusieurs reprises par la meneuse du jeu et ses comparses. Citons les mises en garde « Et gardés que ne me mentés » (v. 108) et «bien li demande / Qu'ele l'en die verité » (v. 146-147), et leur acceptation «Certes, je n'en mentirai mie » (v. 130). 
L'imitation de la confession fonctionne à tel point que le narrateur désigne la meneuse sous le terme «prestres » pendant toute la durée du jeu !

Parce qu'il repose sur une parodie du rituel chrétien de la confession, ce jeu entraine nécessairement un renversement des valeurs. Le passage du rituel religieux au divertissement profane est marqué par l'obscénité du langage. Nous remarquons ainsi dans l'extrait plusieurs calembours licencieux. Le premier, qui s'inscrit dans un échange entre le « confesseur » et l'une des dames, repose sur la paronymie entre « coupe » et « crupe ».

« Une autre revient maintenant,

A la destre main [bat] sa coupe.

- "Douche suer, mais batés la crupe :

$\mathrm{Ki}$ vous fait faire les pechiés

Dont vostre cors est entechiés !” " (v. 122-126)

Le passage du mot «coupe», attaché à la responsabilité morale du chrétien, au mot « crupe » qui désigne de façon imagée et triviale une partie du corps humain, souligne la tension à l'œuvre dans ce lai entre idéal, religieux et/ou courtois selon les passages, et réalité obscène. Un second calembour fondé sur la polysémie du mot « coup » est perceptible quelques vers plus loin :

« - "Dites, dame, comment a non,

Cil ki de vo cuer a le don?"

- "C'est cil dont li païs resonne.

On le doit nommer quant il tonne :

Ja puis ne carra cos en l'estre."

- "Fali avés", che dist li prestre !

"Vous l'avés nommé pluisours fois ;

Onques por çou n'eüstes defois

Que li caus sour vous ne kaïst !

Ja li nons ne vous garesist,

Mais ne fu pas brisiés li caus."

- "Dex me ramaint a iteus caus,

Ne plainge pas la [bon] fuison!"» (v. 179-191) 
Coups de foudre, au sens propre et peut-être au sens figuré ${ }^{7}$, et coups de reins se répondent ici et tirent la confession vers l'obscénité. Enfin le terme même «confesse » peut prêter à confusion. Il est possible de le lire lui aussi comme un calembour scabreux. Cette hypothèse est d'autant plus envisageable qu'une des dames le prononce en riant (v. 104-106). Ainsi, à travers les jeux de mots licencieux, le comique met en évidence d'une part le renversement de la morale chrétienne, puisque sous prétexte de confesser leurs péchés les dames font l'éloge de l'adultère, d'autre part le renversement de la morale courtoise, l'exaltation de l'amour absolu cédant la place à l'évocation du plaisir physique et de la polygamie.

\section{La transgression des règles du jeu courtois}

Paradoxalement, la fausse confession dévoile la vérité cachée et l'observation des règles de ce jeu d'imitation fait apparaître la transgression des règles du jeu courtois. L'amour courtois est en effet conçu dans ce lai comme un jeu, au moins jusqu'au dénouement tragique. Certes, à aucun moment l'amour n'est explicitement assimilé à un divertissement, par l'intermédiaire par exemple d'une comparaison ou d'une métaphore. Les arguments qui nous permettent de voir en l'amour courtois un jeu amoureux et littéraire se trouvent dans le traitement que l'auteur réserve aux thèmes et aux motifs de la fine amor.

$\mathrm{Au}$ tout début du lai, Ignaure est décrit comme un personnage doté de toutes les qualités du chevalier courtois : renommé pour son courage et son goût pour la musique, c'est un jeune homme au «cuer gent » (v. 43) que «fine amors [...] esprent et alume» (v. 36). Toutefois il transgresse une règle essentielle de la fine amor en multipliant les conquêtes. C'est là le principal reproche que lui font les dames :

\footnotetext{
${ }^{7}$ Toutefois l'emploi métaphorique de l'expression « coup de foudre » pour désigner la naissance soudaine du sentiment amoureux est généralement daté du XVII ${ }^{\mathrm{e}}$ siècle.
} 
« Ignaures, tu nous as bien dechutes

Tant con en sommes aperchutes ;

$\mathrm{Ne}$ t'amerons plus en tel guise.

Car il nous ramembre et avise

Que cel ki mius te plaira

Ert toie, et si te remanra.

Chascune velt son dru avoir. » (v. 341-347)

Pour Ignaure, au même titre que la musique et le tournoi auxquels il excelle, l'amour ne semble qu'un divertissement nécessitant une grande habileté. Et assurément il en maîtrise parfaitement les codes. Le lecteur peut mesurer tout le talent du jeune homme lorsque, pris au piège et menacé de mort par ses amies, il réussit à les amadouer en répondant à chacune par une déclaration d'amour dans un pur style courtois. En voici quelques exemples :

«Dame, je suis li vostre amis,

Et vos hom, et vos chevaliers,

Et de vrai cuer fins et entiers. » (v. 292-294)

« Dames, ja ne serés si crueux

Que vous fachiés si grant pechiet.

S'or avoie l'iaume lachiet

Et fuisse el destrier d'Aquilanche,

L'escu au col, el puing la lanche,

Si descendroie jou ichi

Et me metroie en vo merchi. » (v. 324-330)

L'attitude d'Ignaure est ici d'une grande ambiguïté. Le jeune homme est-il sincère? Si tel est le cas, nous assistons à une très intéressante remise en question de l'amour courtois comme un amour unique et absolu. Mais si Ignaure est l'«omme faus, con trahitres et desloiaus» que condamnent les dames (v. 283-284), les formules stéréotypées qu'il emploie se révèlent absolument vides de sens et l'amour courtois apparaît comme un jeu rhétorique, avant même d'être un jeu érotique.

Cette conception de l'amour courtois comme jeu littéraire n'est peut-être pas seulement celle du chevalier ; elle pourrait bien être partagée 
par l'auteur. L'empathie de Renaut pour son personnage semble confirmer cette hypothèse. À aucun moment, en effet, nous ne relevons la moindre condamnation du comportement d'Ignaure par le narrateur. Les seules véritables critiques sont émises par les dames et par le losengier qui le dénoncera aux maris. Ignaure incarne en outre une figure de poète. Son goût prononcé pour la musique et son surnom de Lousignol évoqués au début du lai (v. 30-37), ainsi que la facilité avec laquelle il manie le langage, tendent à faire du chevalier un double de l'auteur.

Par ailleurs, Renaut ne cesse de tourner en dérision la littérature courtoise. Le parallèle avec son personnage peut être poursuivi. En multipliant les déclarations d'amour, Ignaure revendiquait son statut d'amoureux courtois et, dans le même temps, le mettait en péril. De la même façon, l'auteur se présente comme un poète courtois, mais la multiplication des preuves de sa courtoisie éveille les soupçons. Dans le prologue et dans l'épilogue de son œuvre, Renaut prétend avoir écrit un lai, dont les motivations sont, comme le veut la tradition, l'édification des loyaux amants et le souvenir de sa dame. Pourtant l'obscénité assumée de certains passages remet en question l'appartenance du texte au genre du lai pour le rapprocher de celui du fabliau. De plus l'emploi des topoi courtois, par leur surabondance, implique une lecture ironique du texte. Lors du jeu de la confession, par exemple, nous voyons la quatrième dame embrasser son anneau en écoutant un rossignol (v. 163-166). Le chant du rossignol, l'anneau gage d'amour et le baiser sont des lieux communs de la littérature courtoise, mais l'accumulation de ces trois indices en une seule image est excessive et donne une dimension parodique à l'extrait.

Ainsi, la scène de la fausse confession est un moment clef du Lai d'Ignaure, d'un point de vue aussi bien narratif qu'esthétique. Le respect des règles du jeu initié par les dames met en effet en évidence le 
détournement des règles de la courtoisie à plusieurs niveaux. Il s'agit d'abord de la tricherie d'un personnage que sa sensualité pousse à transgresser les lois de la fine amor. Il s'agit surtout de la ruse d'un auteur qui, faisant du texte même son terrain de jeu, détourne les codes de la littérature courtoise pour mieux déjouer les attentes de son lecteur. 